\title{
Concept formation and utilization in the presenc. of irrelevant visual stimulation
}

ED M. EDMONDS and MARVIN R. MUELLFR, Augusta College, Augusta, Ga. 30904

Two experiments assessed the effects of two levels of stimulus redundancy and three levels of irrelevant visual stimulation on performance in a successivediscrimination task and in a reproduction task. The results indicated that increases in redundancy facilitated performance in the reproduction task but had no appreciable effect on performance in the successive-discrimination task. Performance significantly decreased in both tasks as the amount of irrelevant stimulation increased. These findings are discussed in terms of the different strategies that appeared to underlie performance in the iwo tasks.

A number of studies (e.g., Edmonds \& Mueller, 1967a, b; Edmonds, Mueller, \& Evans, 1966) have utilized patterns generated by a computer program, VARGUS 7 (Evans, 1967), to study schematic concept formation in several perceptual tasks. The schema in these patterns is composed of particular column-height sequences favored by the transitional probabilities of a seven-element Markov process. Constraint redundancy is determined by the magnitude of the probabilities associated with the schematic sequences and so can be manipulated independently of the schema. The schema itself can be independently manipulated by varying the favored or most probable column-height sequence. In fact, a population of schemata (column-height sequences) can be defined and sampled. In addition, the pattern-generation procedure allows construction of nonsense forms to have schemata that have been provided neither by experience nor by heredity.

In a series of experiments designed to investigate mixed-schema learning, Edmonds \& Mueller (1967a) found that, in a modified oddity task, Ss readily learned to distinguish among several schemata without knowledge of results. Edmonds \& Evans (1966) and Edmonds \& Mueller (1967b) further demonstrated that, in both a single-schema and a mixed-schema reproduction task, learning not only occurred in a rather spontaneous manner, but could be predicted quite accurately with new Ss and with patterns randomly selected from a different population (schema).
The schema-recognition process evidenced in these studies appears to be identical to concept recognition or to the recognition of equivalence classes. The extreme effectiveness of schema learning is demonstrated by the ability of $S s$ to assign instances to their appropriate schema family without any external source of information. This formation of equivalenco classes reduces the amount of information that must be processed, since all members of one class can be encoded with the same schema. For example, once a schema representing human faces is learned, a particular human face may be recognized by processing only the information in that face that deviates from the "human-face" schema.

One problem, however, that has received relatively little attention concerns the effects of visual noise on the recognition of redundant stimuli. Although a number of investigators (e.g., Attneave \& Arnoult, 1956; Posner, 1964) have studied the properties of distorted forms, the experiments conducted by Rappaport (1957) are especially pertinent to this problem. He investigated the effects of two types and four levels (amounts) of redundancy on the ease with which forms perturbed by noise are recognized. When background noise was present, redundancy facilitated rapid recognition. However, when background noise was absent, an increase in redundancy was accompanied by slower recognition. There were no significant differences between the two types of redundancy in the noise-free situation.

Rappaport attributed the lack of differences between the two types of redundancy in the simultaneousrecognition test to the nonutilization of redundant features of a stimulus by $S$ when external noise was present. He believed that the longer sorting times in the noise-free situation were due to the difficulty of discriminating the smaller details in the figures that were more redundant. Edmonds, Walker, \& Evans ${ }^{1}$ found that, in the noise-free situation, sedundancy facilitated performance in tasks (reproduction) imposing memory requirements but was detrimental to performance in a successive-discrimination task. These results, in conjunction with Rappaport's findings, thus indicate that Ss do not appear to use redundant cues in either a simultaneous-recognition task or a successive-discrimination task but only in tasks involving substantial memory requirements.

The present study, using quantified stimuli and an improved method for producing visual noise, was designed to assess more precisely the effects of different levels of nojse and redundancy on schematic concept utilization in the successive-discrimination task (Experiment 1) and in the reproduction task (Experiment 2). A fact that should be emphasized is that an increase in redundancy produces an increase in the probability of a certain column-height sequence (schema). Consequently, an increase in redundancy at a particular noise level should benefit performance, since $S$ would be progressively better able to learn and to utilize the schema. This supposition particularly holds for the reproduction task, where the use of a schema permits a considerable reduction in the amount of information that must be stored to remember a pattern. The successive-discrimination task imposes a less severe memory requirement than does the reproduction task because a particular pattern does not have to be completely remembered to be selected correctly from among similar patterns. In fact, as noted above, performance in this type of perceptual task may be rather independent of level of redundancy, since Ss may need only to utilize the nonredundant aspects of the stimuli for successful differentiation.

\section{EXPERIMENT 1}

Subjects

The Ss were 60 undergraduate students enrolled in psychology courses at Augusta College. They were assigned randomly to the six treatment combinations.

\section{Patterns}

VARGUS 7 was used to generate 12-column $33 \%$ and $67 \%$ redundant patterns containing the same schema. These patterns served as input to another computer program, VARGUS 6, which accepts patterns in black and white and transmits them to output patterns with any desired level of noise. In this context, noise means that every cell is transmitted with a specified probability of being changed to its opposite state. In VARGUS 6, the probability of error is the same for all cells. The probability levels for correct transmission were $.75, .85$, and 1.00 . For the 1.00 probability (no noise), VARGUS 6 and VARGUS 7 patterns were identical.

\section{Task and Procedure}

Each $\mathrm{S}$ was given a 30-page booklet representing the appropriate noise level. Each odd page of the booklet contained a different VARGUS 7 pattern. Each even 
page of the booklet contained the VARGUS 6 test pattern, corresponding to the preceding VARGUS 7 pattern, along with five similar VARGUS 6 patterns (confusion patterns). The Ss were allowed $15 \mathrm{sec}$ to view each VARGUS 7 pattern. They were then given $30 \mathrm{sec}$ to turn to the next page of the booklet and to select the distorted test pattern from among the five confusion patterns. The position of the test pattern was determined randomly for each page. No knowledge of results was given.

Results

Using number of patterns identified correctly as the responses variable, a two-way analysis of variance, Redundancy $(33 \%, 67 \%)$ by Noise $(1.00, .85, .75)$, indicated that the noise main effect was significant $[F(1,24)=3.28, p<.01]$. The redundancy main effect and the interaction were not significant. The results of the Tukey (a) procedure, which was used to test the differences between the means of the three noise levels, showed that as noise increased, performance significantly decreased.

\section{EXPERIMENT 2} Subjects

The Ss were 60 undergraduates (different from Experiment 1) enrolled in psychology courses at Augusta College.

Task and Procedure

The patterns used in Experiment 1 were also used in this experiment. Each $S$ reproduced 15 patterns in the appropriate condition. A Kodak projector exposed each of the VARGUS 6 patterns, mounted on a $2 \times 2$ in. slide, onto a white screen for $25 \mathrm{sec}$. After each exposure, $\mathrm{S}$ turned to a sheet in a mimeographed booklet where the corresponding VARGUS 7 pattern was printed with three columns omitted. The Ss were given $45 \mathrm{sec}$ to draw the three omitted columns in each pattern. The intertrial interval was $15 \mathrm{sec}$. Since the nature of this task precluded schema learning, Ss received reproduction pretraining to acquaint them with the schematic or redundant aspects of the stimuli. In pretraining, Ss were given 15 reproduction trials with patterns of the same level of redundancy as they were to encounter in the experimental task.

\section{Results}

An analysis of variance based on the number of pattern columns reproduced correctly in the testing phase indicated that the redundancy and noise main effects were significant $(p<.01)$. The Redundancy by Noise interaction was not significant. An individual comparison showed that the $67 \%$ redundant patterns were reproduced significantly $(\mathrm{p}<.01)$ better than the $33 \%$ redundant patterns. Comparisons among noise levels indicated that as noise increased, performance significantly decreased $(\mathrm{p}<.05$ for all comparisons).

\section{DISCUSSION}

The results may be summarized as follows: (1) Increases in the levels of redundancy used in the present study facilitate performance in the reproduction task but have no appreciable effect on performance in the successive-recognition task; (2) performance significantly decreases in both the discrimination and reproduction tasks as noise increases at each redundancy level.

The results of these experiments suggest that different strategies underlie performance in the two tasks. In the reproduction task, where $S$ is required to store all of the information contained in the patterns, schematic or redundancy encoding allows a considerable reduction in the information necessary to effect reproduction, since only those elements that deviate from the schema need be remembered. Consequently, as the patterns become more redundant, less information must be processed in order to perform the task successfully.

Increases in redundancy do not appreciably affect discrimination performance, since $S$ can simply use the distinctive aspects of the stimuli for discrimination. It should be pointed out, however, that substantial increases in redundancy would produce sets of patterns that are highly similar and more difficult to discriminate than are patterns possessing low redundancy. Moreover, patterns that contain no similar attributes $(0 \%$ redundant) should be discriminated better than redundant patterns that are relatively similar (see Edmonds, Walker, \& Evans, 1969).

The results indicate that visual noise affects discrimination and reproduction performance quite similarly. Increasing the amount of irrelevant stimulation reduces the capability of $S$ to utilize the distinctive aspects of the stimuli, which are the features necessary for successful performance in both of the tasks (assuming redundancy encoding has occurred in the memory task).

These experiments provide information concerning the strategies that humans appear to use in two perceptual tasks and indicate the detrimental effects of stimulus distortion on these strategies. Further research should seek to specify more systematically the relationship between amounts and/or types of visual noise on performance in a variety of tasks imposing differential memory requirements.

\section{REFERENCES}

ATTNEAVE, F., \& ARNOULT, M. D. The quantitative study of shape and pattern perception. Psychological Bulletin, 1956, 53, 452-471.

EDMONDS, E. M., \& EVANS, S. H. Schema learning without a prototype. Psychonomic Science, 1966, 5, 247-248.

EDMONDS, E. M., \& MUELLER, M. R. Schema discrimination without external reinforcement. Psychological Reports, 1967a, 24, 436-438.

EDMONDS, E. M., \& MUELLER, M. R. Prediction of mixed schema learning in a reproduction task. Psychonomic Science, 1967b, 8, 533-534.

EDMONDS, E. M., MUELLER, M. R., \& EVANS, S. H. Effects of knowledge of results on mixed schema discrimination. Psychonomic Science, 1966, 6, 377-378.

EVANS, S. H. VARGUS 7: Computed patterns from Markov processes. Behavioral Science, 1967, 12, 323-328.

POSNER, M. I. Uncertainty as a predictor of similarity in the study of generalization. Journal of Experimental Psychology, 1964, 68, 113-118.

RAPPAPORT, M. The role of redundancy in the discrimination of visual forms. Joumal of Experimental Psychology, 1957, 53, 3-10. NOTES

1. Edmonds, E. M., Walker, D. W., \& Evans, S. H. Pattern recognition as a function of constraint redundancy, stimulus channel capacity, and exposure time. In preparation.

2. Supported by OE Grant 8-D-039 to senior author. 